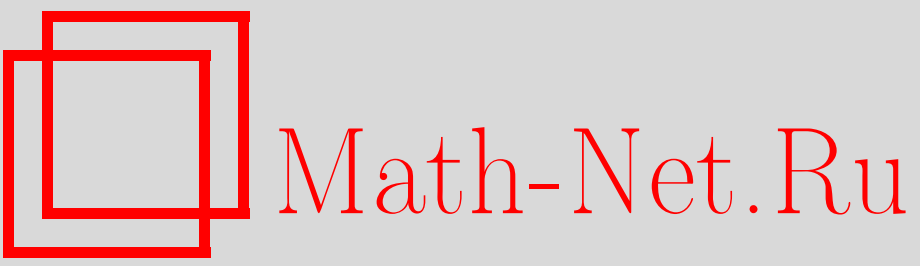

Н. Г. Мощевитин, О наилучших совместных приближениях, УМН, 1996, том 51, выпуск 6, 213-214

DOI: https://doi.org/10.4213/rm1033

Использование Общероссийского математического портала Math-Net.Ru подразумевает, что вы прочитали и согласны с пользовательским соглашением

http://www.mathnet.ru/rus/agreement

Параметры загрузки:

IP : 18.209 .158 .208

26 апреля 2023 г., 12:42:27 


\title{
О НАИЛУЧШИХ СОВМЕСТНЫХ ПРИБЛИЖЕНИЯХ
}

\author{
Н. Г. МощЕвитин
}

\section{1. Введение и формулировки.}

Рассматривается набор вещественных чисел $\alpha=\left(\alpha_{1}, \ldots, \alpha_{s}\right) \in \mathbb{R}^{s}$. Наилучиим совместным приближсением (сокращенно н.п.) к набору $\alpha$ называется целая точка $\zeta=\left(p, a_{1}, \ldots, a_{s}\right) \in$ $\mathbb{Z}^{s+1}$ такая, что

$$
\begin{gathered}
D(\zeta)=\max _{j=1, \ldots, s}\left|p \alpha_{j}-a_{j}\right|<\max _{j=1, \ldots, s}\left|q \alpha_{j}-b_{j}\right|, \\
\forall q: 1 \leqslant q \leqslant p ; \quad \forall\left(b_{1}, \ldots, b_{s}\right) \in \mathbb{Z}^{s} \backslash\left\{\left(a_{1}, \ldots, a_{s}\right)\right\} .
\end{gathered}
$$

Всюду далее в этом пункте все $\alpha_{j}$ иррациональны. Все н.п. к $\alpha$ можно расположить в виде бесконечной последовательности $\zeta^{\nu}=\left(p^{\nu}, a_{1}^{\nu}, \ldots, a_{s}^{\nu}\right), \nu=1,2, \ldots$, так, что $p^{1}<\cdots<p^{\nu}<$ $p^{\nu+1}<\cdots$ и $D\left(\zeta^{1}\right)>\cdots>D\left(\zeta^{\nu}\right)>D\left(\zeta^{\nu+1}\right)>\cdots$. Положим

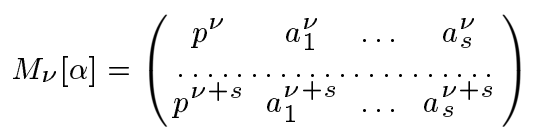

и через $\operatorname{rk} M_{\nu}[\alpha]$ будем обозначать ранг квадратной матрицы $M_{\nu}[\alpha]$. Определим натуральное число $R(\alpha), 2 \leqslant R(\alpha) \leqslant s+1$, из условия

$$
\begin{gathered}
R(\alpha)=\min \left\{n: \text { найдется решетка } \Lambda \subseteq \mathbb{Z}^{s+1}, \operatorname{dim} \Lambda=n\right. \\
\left.\quad \text { и натуральное } \nu_{0} \text { такое, что } \zeta^{\nu} \in \Lambda \forall \nu>\nu_{0}\right\} .
\end{gathered}
$$

Величина $\operatorname{dim}_{\mathbb{Z}} \alpha$ определяется как максимальное количество чисел $\alpha_{i_{1}}, \ldots, \alpha_{i_{m}}$, которое можно выбрать из набора $\left(\alpha_{0}=1, \alpha_{1}, \ldots, \alpha_{s}\right) \in \mathbb{R}^{s+1}$ так, чтобы они были линейно независимы над кольцом целых чисел $\mathbb{Z}$.

УтвеРЖДЕнИЕ 1. Пусть $s=1$. Тогда для любого $\nu$ выполнено $\operatorname{det} M_{\nu}[\alpha]= \pm 1$ (m.е. $\left.\operatorname{rk} M_{\nu}[\alpha]=2 \quad \forall \nu\right)$.

УТВеРЖДЕНИЕ 2. При произвольном $s \geqslant 1$ выполнено $R(\alpha)=\operatorname{dim}_{\mathbb{Z}} \alpha$.

УТВЕРЖДЕНИЕ 3. Пусть $s=2$ и $\alpha_{1}, \alpha_{2}$ линейно независимы вместе с единицей над $\mathbb{Z}$. Тогда найдется бесконечно много натуральных $\nu$ таких, что $\operatorname{rk} M_{\nu}[\alpha]=3=\operatorname{dim}_{\mathbb{Z}} \alpha$ (т.е. для бесконечно многих $\nu$ выполнено $\left.\operatorname{det} M_{\nu}[\alpha] \neq 0\right)$.

Утверждения 1, 2, 3 общеизвестны и легко проверяются. Следующая теорема представляет собой новьй результат.

Теорема. Пусть $s \geqslant 3$. Тогда существует континуальное семейство наборов $\alpha$ чисел $\alpha_{1}, \ldots, \alpha_{s}$, линейно независимых вместе с единицей над $\mathbb{Z}$, таких, что $\mathrm{rk} M_{\nu}[\alpha] \leqslant 3$ $\forall \nu \in \mathbb{N} .\left(\right.$ В частности, для всех $\nu$ выполнено $\operatorname{det} M_{\nu}[\alpha]=0$, хотя $\left.\operatorname{dim}_{\mathbb{Z}} \alpha=s+1.\right)$

ЗАмечАния. 1) Сформулированная теорема представляет собой контрпример к гипотезе Лагариса [1].

2) На возможность получения результатов типа данной теоремы автору указал Н. П. Долбилин. В связи с этим автор предлагает называть конструкцию из доказательства теоремы спиралью Долбилина.

3) Аналогичные результаты для линейных форм наименее уклоняющихся от нуля были получены автором в [2].

Работа выполнена при финансовой поддержке Российского фонда фундаментальных исследований (грант № 96-01-00378). 


\section{2. Лемма.}

Рассматривается евк лидово пространство $\mathbb{R}^{s+1}$, отнесенное к декартовой системе координат $\left(x, y_{1}, \ldots, y_{s}\right)$. Через $\ell$ мы будем обозначать луч, исходящий из начала координат в полупространство $x>0$. Для луча $\ell$ и положительного числа $\varepsilon$ конус $K_{\varepsilon}(\ell)$ определим состоящим из всех лучей $\ell^{\prime}$ таких, что угол между $\ell$ и $\ell^{\prime}$ меньше $\varepsilon$. Для точки $\xi$ из полупространства $x>0$ обозначим $\ell(\xi)$ луч $[0 ; \xi)$. Будем называть плоскость $\pi \subseteq \mathbb{R}^{s+1}$ вполне рациональной, если содержащаяся в ней решетка $\Lambda=\pi \cap \mathbb{Z}^{s+1}$ такова, что $\operatorname{dim} \Lambda=\operatorname{dim} \pi$. Наилучшим приближением к лучу $\ell$ с направляющим вектором $\left(1, \beta_{1}, \ldots, \beta_{s}\right)$ мы будем называть точку $\zeta \in \mathbb{Z}^{s+1}$, являющуюся н.п. к набору $\beta$ в смысле, определенном выше. Если считать, что $\beta_{j}$ при каждом $j$ не есть полуцелое число, то последовательность н.п. $\zeta^{\nu}=\left(p^{\nu}, a_{1}^{\nu}, \ldots, a_{s}^{\nu}\right), p^{1}<\cdots<p^{\nu}<p^{\nu+1}<\cdots$ к лучу $\ell$ (конечная или бесконечная, в зависимости от того, имеется ли на луче $\ell$ целая точка, отличная от начала координат, или нет) определена однозначно, и мы будем обозначать ее $\mathscr{B}(\ell)=$ $\left\{\zeta^{1}, \zeta^{2}, \ldots, \zeta^{\nu}, \ldots\right\}$. Также мы будем использовать обозначение $\mathscr{B}_{k}^{t}(\ell)=\left\{\zeta^{k}, \zeta^{k+1}, \ldots, \zeta^{t}\right\}$.

Лемма. Пусть $\Lambda=\mathbb{Z}^{s+1} \cap \pi$-решетка во вполне рациональной плоскости $\pi$ размерности $\operatorname{dim} \pi \geqslant 2$. Пусть $\zeta \in \Lambda$ - целая точка такая, что

$$
\mathscr{B}(\ell(\zeta))=\left\{\zeta^{1}, \zeta^{2}, \ldots, \zeta^{\tau}, \ldots, \zeta^{t}\right\},
$$

$\zeta^{t}=\zeta$, и, кроме того, $\mathscr{B}_{\tau}^{t}(\ell(\zeta))=\left\{\zeta^{\tau}, \ldots, \zeta^{t}\right\} \subset \Lambda \subset \pi$. Пусть, вдобавок, $D\left(\zeta_{\tau}\right)<D(\xi)$ для любой челой точки $\xi$, не принадлежсащей плоскости $\pi$. Тогда найдется $\varepsilon>0$ такое, что для любого луча $\ell^{\prime} \subset K_{\varepsilon}(\ell)$ выполняется 1) $\mathscr{B}\left(\ell^{\prime}\right) \supset \mathscr{B}(\ell)$; 2) последовательность н.п. к лучу $\ell^{\prime}$, расположенных между приближениями $\zeta^{\tau}$ и $\zeta^{t}$ (содержащая по крайней мере все приближения из $\left.\mathscr{B}_{\tau}^{t}(\ell(\zeta))\right)$ челиком лежит во вполне рачиональной плоскости $\pi$.

Доказательство леммы основано на следующих соображениях.

1. При небольшом шевелении $\ell^{\prime}$ луча $\ell$ начальные н.п. к $\ell$ остаются н.п. и к $\ell^{\prime}$, хотя кроме этих наилучших приближений, вообше говоря, могут появиться новые наилучшие приближения.

2. Небольшое шевеление луча $\ell$ не позволяет появиться между приближениями $\zeta^{\tau}$ и $\zeta^{t}$ наилучшим приближениям, не находящимся во вполне рационалной плоскости $\pi$.

\section{3. Схема доказательства теоремы.}

3.1. Многократно применяя лемму, строим вполне рациональные плоскости $\pi_{1}, \rho_{1}, \pi_{2}, \rho_{2}, \ldots$, $\pi_{s}, \rho_{s}$ размерностей $\operatorname{dim} \pi_{j}=2, \operatorname{dim} \rho_{j}=3$ и луч $\ell=\ell(\zeta), \zeta \in \pi_{s}$, так, чтобы, во-первых, $\pi_{j}, \pi_{j+1} \subset \rho_{j}$, во-вторых,

$$
\mathscr{B}(\ell)=\left\{\zeta^{1}, \ldots, \zeta^{\tau_{1}}, \zeta^{\tau_{1}+1}, \ldots, \zeta^{t_{1}}, \zeta^{t_{1}+1}, \ldots, \zeta^{\tau_{2}}, \ldots, \zeta^{\tau_{s}+1}, \ldots, \zeta^{t_{s}}\right\},
$$

где $\zeta^{t_{s}}=\zeta, t_{0}=1$, причем $t_{j}-\tau_{j} \geqslant s+1, \tau_{j}-t_{j-1} \geqslant s+1 \forall j$ и $\zeta^{\tau_{j}+1}, \ldots, \zeta^{t_{j}} \in \pi_{j} \forall j$, $\zeta^{t_{j}+1}, \ldots, \zeta^{\tau_{j+1}} \in \rho_{j} \forall j$, и, в-третьих, $\mathscr{B}(\ell)$ (равно как и объединение $\bigcup_{j=1}^{s} \rho_{j}$ ) не лежало бы ни в каком $s$-мерном подпространстве пространства $\mathbb{R}^{s+1}$.

3.2. Многократно применяя процедуру пункта 3.1, строим последовательность лучей $\ell^{k}=\ell\left(\xi^{k}\right), \xi^{k} \in \mathbb{Z}^{s+1}, k=1,2, \ldots$, так, что для каждого луча $\ell^{k}$ множество н.п. $\mathscr{B}\left(\ell^{k}\right)$ состоит из $k$ штук подряд идущих блоков, каждый из которых обладает свойствами, описанными выше в пункте 3.1 .

Предельный луч для последовательности лучей $\ell^{k}$ и будет задавать числа, удовлетворяющие условию теоремы: все наборы подряд идущих $s+1$ н.п. для них в силу построения и леммы будут лежать в некоторых дву- или трехмерных плоскостях, а утверждение 2 и опять же процедура построения обеспечивают линейную независимость.

\section{СПИСОК ЛИТЕРАТУРЫ}

[1] Lagarias J. S. // Pac. J. Math. 1982. V. 102. № 1. P. 61-88. [2] Мощевитин Н. Г. О геометрии наилучших приближений // Докл. РАН. (в печати). 